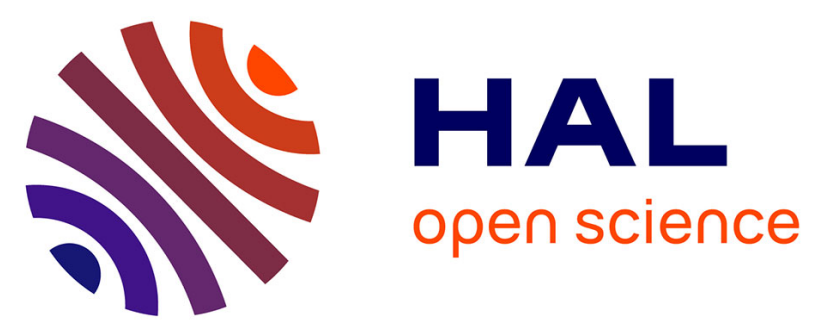

\title{
Global View on Dose Rate Effects in Silica-Based Fibers and Devices Damaged by Radiation-Induced Carrier Trapping
}

Franck Mady, Mourad Benabdesselam, Jean Bernard Duchez, Yasmine Mebrouk, Sylvain Girard

\section{To cite this version:}

Franck Mady, Mourad Benabdesselam, Jean Bernard Duchez, Yasmine Mebrouk, Sylvain Girard. Global View on Dose Rate Effects in Silica-Based Fibers and Devices Damaged by RadiationInduced Carrier Trapping. IEEE Transactions on Nuclear Science, 2013, 60 (6), pp.4341-4348. 10.1109/TNS.2013.2282410 . ujm-01011687

\section{HAL Id: ujm-01011687 https://hal-ujm.archives-ouvertes.fr/ujm-01011687}

Submitted on 26 Apr 2016

HAL is a multi-disciplinary open access archive for the deposit and dissemination of scientific research documents, whether they are published or not. The documents may come from teaching and research institutions in France or abroad, or from public or private research centers.
L'archive ouverte pluridisciplinaire HAL, est destinée au dépôt et à la diffusion de documents scientifiques de niveau recherche, publiés ou non, émanant des établissements d'enseignement et de recherche français ou étrangers, des laboratoires publics ou privés. 


\title{
Global View on Dose Rate Effects in Silica- Based Fibers and Devices Damaged by Radiation-Induced Carrier Trapping
}

\author{
Franck Mady, Mourad Benabdesselam, Jean-Bernard Duchez, Yasmine Mebrouk and Sylvain Girard
}

\begin{abstract}
We give a novel and very general treatment to a standard model describing dose rate effects in systems damaged by carrier trapping. This model is well adapted for optical fibers, but the lessons we draw may also be helpful to discuss DRE in electronic devices. By highlighting the few determinant ratios of physical parameters that govern the system behavior, we clarify when, how and how much dose rate effects affect trap filling and radiation-induced degradation. Critical dose rate, marking the demarcation between low and high dose rate regimes, is also estimated as a function of these parameters. Taking this step back is important to enlighten contradictory results reported on DRE behaviors. The dose and dose rate dependencies of trap filling measured on silica optical fibers, as well as the critical dose rate, are successfully reproduced with a single set of a few adjustable physical parameters.
\end{abstract}

Index Terms - Carrier trapping, Dose rate effects, Modeling, Optical Fibers, Total dose effects

\section{INTRODUCTION}

Silica optical fibers and fiber-based sensors are of major interest in a variety of applications in space or nuclear backgrounds. Embedded optical inter-satellite links (OISL) or remote sensing (LIDAR) could e.g. benefit from high power fiber laser sources based on erbium or ytterbium-doped fiber amplifiers that offer the advantages of reduced weight, size, power consumption, cost, and greater efficiency compared with solid-state lasers. The development of the fiber technology is however limited by radiation-induced darkening, a considerable excess optical loss that develops across the UV, visible and near-IR spectral ranges. By affecting pump and

Manuscript revised 09/11/2013, Accepted 09/11/2013.

This work partly fall within the framework of the HAPoLO (098-AO112408) and PARADYSIO (ANR-11-JS04-007) projects supported by the EADS Foundation and the French Research National Agency (ANR).

F. Mady, M. Benabdesselam, J-B. Duchez and Y. Mebrouk were with the University of Nice Sophia Antipolis, Laboratoire de Physique de la Matière Condensée, CNRS UMR 7336, Faculté des Sciences, Parc Valrose, 06108 Nice, France (e-mail: franck.mady@unice.fr).

S. Girard was with the University Jean Monnet, Laboratoire Hubert Curien, CNRS UMR 5516, 18 rue du Professeur Benoît Lauras, 42000 Saint Etienne, France. signal wavelengths, it has dramatic consequences on the performance of fiber-based systems. The design and test of radiation-tolerant fibers have to cope with the dose rate dependency of darkening. This question is crucial in spacebased applications where the radiation resistance is assessed by accelerated tests, conducted at a much higher dose rate than those encountered in actual operational conditions. Beyond the understanding of dose rate effects (DRE), the challenge is to set proper accelerated test protocols.

DRE have been a major concern for a long time in electronics. Degradation of bipolar junction transistors (BJTs) is for instance very dose rate sensitive [1,2]. At any given dose, these components most often present higher degradation levels at low than at high dose rates, this feature being termed "Enhanced Low Dose Rate Sensitivity" (ELDRS). An ELDRS explanation based on a trap model was proposed in [3]: assuming that degradation results from hole trapping on gap levels, and based on a simple set of rate equations, ELDRS was argued to result from the competition between trapping and recombination in the presence of a high concentration of recombination centers due to the poor oxide quality. The same group proposed the "dose rate switching" method to conduct accelerated tests on BJTs and related technologies [4].

Evidence for ELDRS in an erbium-doped fiber amplifier (EDFA), the most common type of fiber amplifier, was also reported [5]. This finding motivated attempts at dose rate switching experiments, as in [6] where a theory in qualitative agreement with data is also proposed. The model of [6] is based on a single rate equation, a single population, and thus poorly accounts for the competition between trapping and recombination. Color centers are generated following a powerlaw dose rate dependent term and relax according to dispersive detrapping kinetics. Dispersion is a key ingredient rather than a refinement in this model because taking classical (nondispersive) detrapping would result in a dose-independent degradation at the low dose rate limit, and in enhanced high dose rate sensitivity only. Trapping-recombination models offer a better universality: they involve several carrier populations and are able to produce ELDRS regardless of the detrapping kinetics.

Similarities between BJTs and EDFA as regards ELDRS and possible routes of accelerated test suggest that electronics and fiber optics may, to a certain extent, receive a joint treatment. Darkening processes in fibers can be cast into trapping-recombination models, as shown by our works $[7,8]$. Numerical treatments, including those of [3], were always 
restricted to a few particular sets of physical parameters and are unable to state clearly when, how, and how much the dose rate affects degradation. Given the general importance of this question, especially for space-based applications, and the contradictory results reported on the dose rate dependency of radiation-induced damage, this work brings a novel perspective and clarifies the conditions making low dose rate enhance or reduce the degradation resulting from carrier trapping. The critical dose rate, marking the demarcation between low and high dose rate regimes is also discussed, based on the defect density and the polarity of trapped carriers notably. Comparison with experimental data on silica optical fibers shows that the model is capable of describing the dose and dose rate dependences as well as the critical dose rate.

\section{The Model and its Global Treatment}

\section{A. General model}

A standard trapping-recombination model is illustrated in Fig. 1 and the related rate equations (1) to (4). Pair generation takes place at a constant rate $g_{0}$ per unit volume and time. Holes generated in the valence band (VB) only undergo capture on recombination centers (RCs). Electrons generated in the conduction band (CB) can be trapped by a set of $M$ discrete trapping levels or recombine with holes on RCs. $n_{\mathrm{c}}$ and $h_{\mathrm{v}}$ are the concentrations of electrons in the CB and of holes in the VB respectively. $N_{k}$ is the concentration of traps $k$ and $n_{k}$ is the electron density on these traps. The trapping coefficient is $\beta_{k}=\sigma_{\mathrm{k}} v_{\text {th }}$ for the $k$ th trap, where $\sigma_{k}$ is the capture cross-section and $v_{\text {th }}$ the thermal velocity in the CB. The recombination coefficient is $\gamma=\sigma_{\mathrm{r}} v_{\mathrm{th}}$ where $\sigma_{\mathrm{r}}$ is the recombination cross-section. The hole trapping coefficient is $\delta$. Hole traps, whose concentration is $H$, serve as RCs and are populated by $h$ trapped holes ( $h$ also sets the RC density).

The evolution of the electron density in the $\mathrm{CB}$ is described by (1) where the right-hand side term represents the generation, trapping and recombination rates respectively:

$d n_{c} / d t=g_{0}-n_{c} \sum_{k=1}^{M} \beta_{k}\left(N_{k}-n_{k}\right)-\gamma n_{c} h$.

The evolution of the trapped electron density is assumed to result from trapping only, as seen in (2):

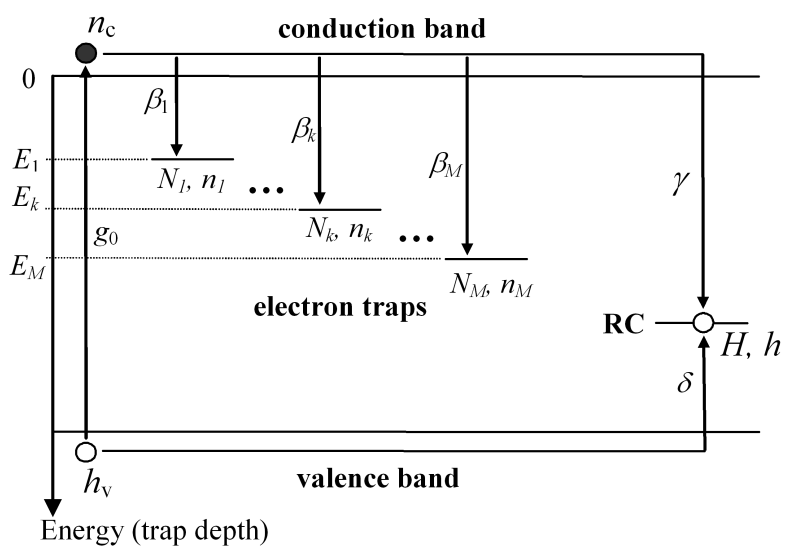

Fig. 1. The energy levels model with its transition parameters. Degradation is associated with electron traps. Hole traps act as the recombination centers. $d n_{k} / d t=\beta_{k} n_{c}\left(N_{k}-n_{k}\right), \quad k=1 . . M$.

The hole population in the VB varies according to the generation and trapping on RC and hence obeys (3):

$d h_{\mathrm{v}} / d t=g_{0}-\delta h_{\mathrm{v}}(H-h)$,

At last, the population of holes on RC evolves due to hole trapping and recombination according to (4):

$d h / d t=\delta h_{\mathrm{v}}(H-h)-\gamma n_{c} h$.

As in [3] for BJTs or [6] for EDFA, this kinetic model describes a spatially homogeneous material, free of carrier flow. In the absence of electric field and population gradients, the electrical neutrality is an inherent property of the material described by (1) to (4). Based on the assumption that the radiation-induced degradation is represented by the amount of trapped carriers, our basic task was to predict the total dose and dose rate dependences of trap filling. This hypothesis is well justified for optical fibers since darkening develops due to the radiation-induced release of free carriers that trap on defects to form the color centers responsible for optical absorption. It is probably insufficient for BJTs whose degradation further involves the migration of protons released upon hole trapping to a $\mathrm{Si} / \mathrm{SiO}_{2}$ interface. Migration would require drift or diffusion, but these phenomena are neither taken into account in (1) to (4), nor in [3].

All trapped states are assumed to be thermally stable during and after irradiation so detrapping is neglected. We thus focus on DRE resulting from the competition between trapping and recombination, the landscape being not clouded by thermal recovery under radiation exposure. The hypothesis of a single $\mathrm{RC}$ is made for simplicity, but it is rather justified for optical fibers where one $\mathrm{RC}$ most often prevails. This is e.g. the case in germanium-doped fibers, where recombination mainly takes place on germanium-related oxygen deficient centers [9], or in ytterbium-doped fibers where ytterbium ions were shown to be the largely predominant RCs [8]. Then, color centers are essentially related to the trapping of carriers whose polarity is opposite to the charge of RCs. Here, we associate degradation with electron trapping only, the RCs being charged by holes. This arbitrary choice does not limit generality since the role of carriers can be freely reversed.

\section{B. Normalization strategy}

A dimensionless formulation is most helpful to clear the formalism from particular values of the physical variables and highlight the few parameter ratios that rationalize the competition between the various transitions at play and hence govern the dose and dose rate dependences. Trap filling is estimated through single trap filling rates $\varphi_{k}\left(0 \leq \varphi_{k} \leq 1\right)$ and the overall trap filling rate $\phi(0 \leq \phi \leq 1)$, defined by:

$$
\begin{aligned}
& \varphi_{k}=n_{k} / N_{k}, \\
& \phi=n / N, \text { with } n=\sum_{k=1}^{M} n_{k} \text { and } N=\sum_{k=1}^{M} N_{k} .
\end{aligned}
$$

These filling rates are not independent, since:

$$
\phi=\sum_{k=1}^{M} \xi_{k} \varphi_{k} \text {, with } \xi_{k}=N_{k} / N \text {. }
$$


The $\xi_{k}$ coefficients define the relative fraction of each trap $k$ and therefore the discrete density of trapping states.

The dose is proportional to the total number $N_{\mathrm{EHP}}$ of electron-hole pairs (EHP) created by irradiation per unit volume. It depends on the irradiation duration $t_{\text {irr }}$ according to $N_{\text {EHP }}=g_{0} \times t_{\text {irr }}$. In the model, $g_{0}$ and $N_{\text {EHP }}$ naturally represent the dose rate and the dose respectively. The latter is measured with respect to the trap density $N$ to give the dimensionless dose coefficient $D=N_{\mathrm{EHP}} / N$. Taking $D<<1$ will thus ensure that traps are kept far below saturation and conversely.

A possible dimensionless form of (1) to (4) is:

$$
\begin{aligned}
& d u_{\mathrm{c}} / d \tau=R-u_{\mathrm{c}} \sum_{k=1}^{M} \alpha_{k} \xi_{k}\left(1-\varphi_{k}\right)-r u_{\mathrm{c}} \varphi_{\mathrm{h}} \\
& d \varphi_{k} / d \tau=\alpha_{k} u_{\mathrm{c}}\left(1-\varphi_{k}\right), k=1 . . M \\
& d u_{\mathrm{v}} / d \tau=R-\theta r u_{\mathrm{v}}\left(1-\varphi_{\mathrm{h}}\right) \\
& d \varphi_{\mathrm{h}} / d \tau=\theta u_{\mathrm{v}}\left(1-\varphi_{\mathrm{h}}\right)-u_{\mathrm{c}} \varphi_{\mathrm{h}} .
\end{aligned}
$$

The populations $n_{\mathrm{c}}$ and $h_{\mathrm{v}}$ have been normalized to the density of traps to give $u_{\mathrm{c}}=n_{\mathrm{c}} / N$ and $u_{\mathrm{v}}=h_{\mathrm{v}} / N$ and $\varphi_{\mathrm{h}}=h / H$ is the RC occupancy rate. The reduced time is $\tau=\gamma N t I$, and $R=g_{0} / \gamma N^{2}$ appears as the dimensionless dose rate coefficient.

The system described by (8) to (11) is fully determined by $M+2$ normalized parameters. Te ratio of the RC concentration to the density of traps, measured by $r=H / N$, and the transition coefficient ratios representing the competition between electron trapping and recombination,

$\alpha_{k}=\beta_{k} / \gamma$, for $k=1 . . M$,

and between hole trapping and recombination

$\theta=\delta / \gamma$.

If we make the convenient assumption that all traps have the same capture cross-section (the same $\beta, \alpha_{k}=\alpha$ ), only three parameters remain: $r, \alpha$ and $\theta$. Then, (9) does not depend on $k$, each single $\varphi_{k}$ obeys the same equation, and traps fill up parallel to one other following a same occupancy rate $\varphi_{k}(\tau)=\phi(\tau)$. Therefore the distribution of trapped electrons parallels the distribution of traps $\left(n_{k}=\xi_{k} \times n\right)$ and the problem is reduced to a single trap system $(M=1)$. For the same reason, taking only one RC in Fig. 1 does not mean that the model is strictly restricted to a single type of RC. $H$ and $h$ should be seen as total concentrations related to a collection of RCs having the same cross-sections. The assumption of a single cross-section is not realistic, but it will give the correct trends if cross-sections roughly remain of the same order of magnitude (the single cross-section will be representative of a mean value). If not, the trapping coefficient, trap densities and trap populations should be associated with the averages of most attractive traps only, the other being neglected.

\section{Predicted Dose Rate Effects}

If recombination was neglected in the model of Fig. 1, all the generated electrons would be eventually trapped and the SSOR would necessarily be $n=N_{\text {EHP }}$, i.e. $\phi=D$. Even in the case of trap saturation $(\phi=1)$, trap filling is therefore dose rate independent in the absence of recombination. Obviously, DRE result from the presence of a recombination mechanism competing with trapping. Steady-state occupancy rates (SSORs) left by irradiation can be calculated by solving (8) to (11), first under irradiation (at given $R$ and $D$ ) starting from $\varphi_{k}(0)=0$ for any $k$, and then by calculating the dark relaxation $(R=0)$, right after the end of the radiation exposure, until trap populations reach a constant value (empty bands). Equations (8) to (11) form a set of nonlinear coupled differential equations. It has generally to be solved numerically, even if decisive formal advances can be made if $\theta>>1$ (strong hole trapping) and $r \gg>1$ (much more RC than traps).

\section{A. The case of direct recombination $(\theta>>1$ and $r>>1)$}

Direct recombination, i.e. recombination between an electron from the $\mathrm{CB}$ and a hole in the $\mathrm{VB}$, does not involve any $\mathrm{RC}$. Holes can be both generated and recombined on a donor level in the gap, instead of the VB, provided generation is still unsaturable (dose below the donor exhaustion threshold). This case is rigorously achieved by making $\delta$ and $H$ tend to infinity and corresponds to the limit $\theta \gg>1$ and $r>>1$. Then, the competition between trapping and recombination is solely governed by $\alpha=\beta / \gamma$, the electron trapping to recombination cross-section ratio. This case produces the largest DRE compared with situations with smaller $\theta$ and $r$, i.e. when recombination is additionally limited by a small probability of hole trapping on RC (small $\theta$ ) or/and by a small density of RC (small r). Merging (10) and (11) to consider direct recombination and eliminating $u_{\mathrm{c}}$ by partial integration of (8) and (9) allowed us to calculate the exact SSORs at both limit dose rates $(R \rightarrow 0$ and $R \rightarrow+\infty)$. We obtained for $p=1 . . M$ :

$D=\sum_{k=1}^{M} \xi_{k}\left(1-1 / \alpha_{k}\right)\left[1-\left(1-\varphi_{p}\right)^{\alpha_{k} / \alpha_{p}}\right]-\ln \left(1-\varphi_{p}\right) / \alpha_{p}$

for $R \rightarrow 0$, and

$D=\sum_{k=1}^{M} \xi_{k}\left[1-\left(1-\varphi_{p}\right)^{\alpha_{k} / \alpha_{p}}\right] /\left(1-\varphi_{p}\right)^{1 / \alpha_{p}}$

for $R \rightarrow+\infty$. Given the material parameters $\xi_{k}$ and $\alpha_{k}$ $(k=1 . . M),(14)$ and (15) give the dose $D$ required to achieve a SSOR $\varphi_{p}$ in the $p$ th trap. If traps have the same capture cross section $\left(\alpha_{k}=\alpha\right), \varphi_{k}=\phi$ (same single and global SSORs) and (14) and (15) reduce to:

$$
\begin{aligned}
& D=(1-1 / \alpha) \phi-\ln (1-\phi) / \alpha \text { and } \\
& D=\phi /(1-\phi)^{1 / \alpha}
\end{aligned}
$$

for $R \rightarrow 0$ and $R \rightarrow+\infty$ respectively. These equations must be reversed to get SSORs as a function of the dose $D$. For identical trapping and recombination cross-sections $(\alpha=1)$, e.g., $\phi=1-\exp (-D)$ if $R \rightarrow 0$ and $\phi=D /(1+D)$ if $R \rightarrow+\infty$.

Comparison of (14) and (15), or of simplified forms (16) and (17), shows that SSORs are always higher at the low dose rate limit than at the high one. Hence direct recombination $(\theta>>1$ and $r \gg>1)$ always produces an ELDRS. Its amplitude is negligible for $\alpha \geq 1$, but it increases dramatically as $\alpha$ passes below unity (more efficient recombination). Examples of SSORs calculated with (16) and (17) are plotted in Fig. 2 as a function of the dose for various $\alpha$ values. Plots of SSORs as a function of $R$ (at fixed dose), not shown here, are always found to be sigmoid-like curves, regardless of $\alpha$. 


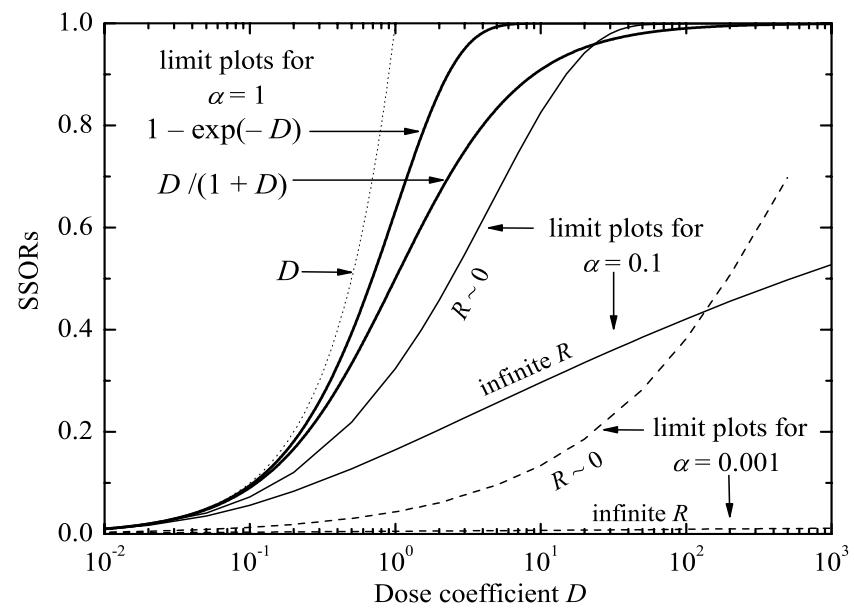

Fig. 2. Examples of steady-state trap occupancy rates left by irradiation for direct recombination and a single trapping cross-section $\left(\beta_{k}=\beta\right)$. Traps are empty if SSORs $=0$ and saturated when SSORs $=1$. The plot SSOR $=D$ locates the highest possible SSOR, obtained in the absence of recombination.

The preceding results can be altered either by a low $\delta$, by a small density of RCs, or by any combination of both effects. To discriminate between each single contribution, we focus on two particular situations in what follows: (i) $\theta<1$ and $r>1$. Then, hole trapping is limited by a small cross-section (small $\delta$ ), but not by RCs which saturate at much higher doses than electron traps; and (ii) $\theta>1$ and $r<1$. Then, the recombination efficiency only suffers from the saturation of RCs occurring from a dose below the saturation threshold of traps. We pay attention in cases where $\alpha<1$ because no noticeable DRE are found for $\alpha>1$. All SSORs were calculated numerically with the Scilab ${ }^{\circledR}$ software.

\section{B. Effect of a weak hole trapping probability $(r>>1, \theta \leq 1)$}

Small hole capture cross-sections deteriorate recombination efficiency by delaying hole trapping. This effect is not significant if the rate of pair creation is much smaller than the rate of hole capture. Therefore SSORs are still given by (14) or its simplified form (16) - if the dose rate tends to zero. Fig. 3 displays the SSOR dose dependence calculated at infinite dose rate for $r=H / N=100, \alpha=\beta / \gamma=0.001$ and several values of $\theta=\delta / \gamma$. Also shown in dashed lines are the plot of (17) representing direct recombination, and the $\mathrm{SSOR}=D=$ $N_{\mathrm{EHP}} / N$ curve setting the maximum trap filling (in the absence of recombination). The $R=0$ limit plot, independent of $\theta$ and given by (16), appears in thin line. Trap filling follows the direct recombination formula (17) at low doses $(D<10 \theta)$ and then grows much faster towards saturation. A crossover thus appears if $\theta<1$ between infinite $R$ plots and the $\theta$-independent one at $R=0$, evidencing the possibility of obtaining a higher trap occupancy at high dose rate. As the ratio $\theta$ between hole trapping and recombination is decreased, hole trapping becomes unlikely and curves move closer the recombinationfree case $(\mathrm{SSOR}=D)$ while the crossover dose is shifted towards small doses. Computed SSORs are plotted against the dose rate coefficient in Fig. 4 for $r=100, \alpha=0.001$ and $\theta=$ 0.001 . In agreement with Fig. 3 , they decrease with increasing $R$ for $D<20$, increase with $R$ otherwise, always following typical S-shaped curves. Variations are well monotonic, except around the crossover dose $(D \sim 20$, see inset).

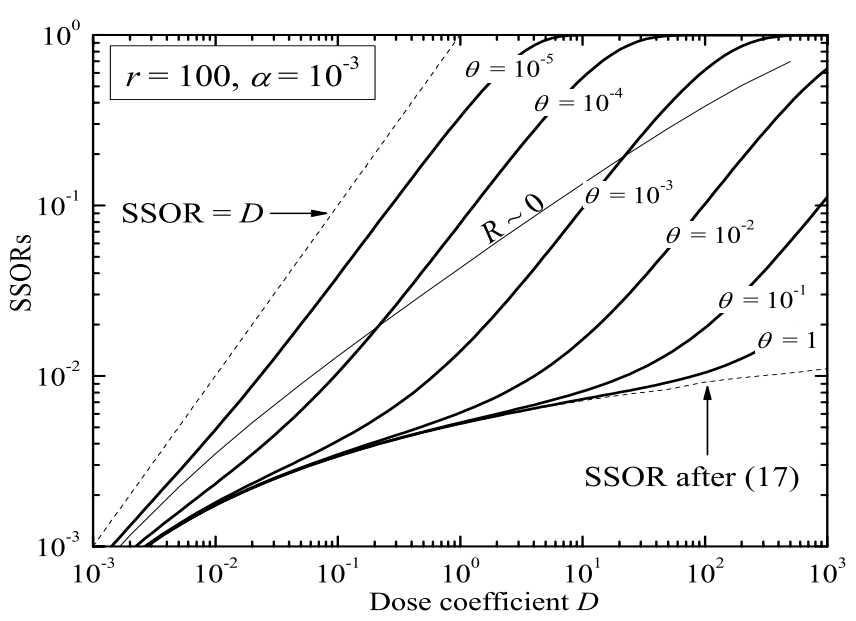

Fig. 3. SSORs at infinite dose rate (solid lines) for $r=100$ and $\alpha=0.001$. Dashed lines are for negligible recombination (upper curve) and direct recombination (lower curve, after (17)); the thin line is obtained when $R$ tends to zero and is given by (16).

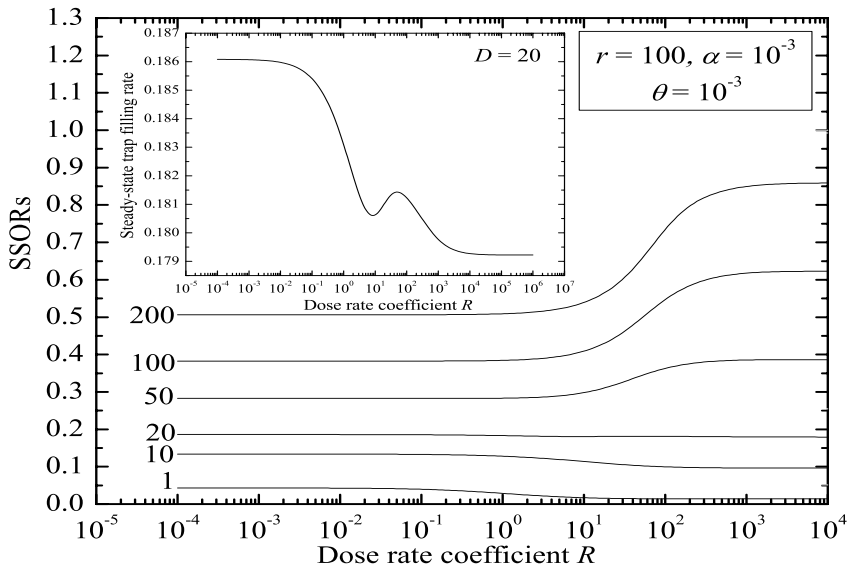

Fig. 4. SSORs as a function of the dose rate coefficient for $r=100$, $\alpha=0.001$ and $\theta=0.001$. Doses $D$ are indicated by plot labels. Inset: focus on the plot $D=20$.

\section{Effect of RC saturation ( $r \leq 1, \theta>>1)$}

In the complementary case, hole capture and recombination efficiency are limited by the saturation of RC due to a small $H$. For $r<1$ (more traps than RCs), this will occur before the saturation of electron traps, i.e. for SSORs significantly smaller than unity. At saturation, the condition $\varphi_{\mathrm{h}}=h / H=1$ leads to the exact expression of the SSOR $\phi=n / N$ for a single electron trapping coefficient, at any dose rate:

$D=\phi-\ln (1-\phi)^{r / \alpha}$.

A sufficient condition to prevent RC saturation is $N_{\mathrm{EHP}}<<H$, or $D<<r$ in dimensionless terms. Occupancy rates are therefore the same as those calculated for direct recombination if $D<<r$, regardless of the dose rate.

SSORs obtained at both dose rate limits for $\theta=100$ $(\delta=100 \times \gamma), r=0.1(N=10 \times H)$, and $\alpha=0.01(\gamma=100 \times \beta)$ are shown by solid lines in Fig. 5. Direct recombination SSORs (16) and (17) are shown in thin lines, and the dashed line is the plot of (18) for saturated RCs. Computed plots well obey the direct recombination model for $D<<r$ at both limits. As $D$ exceeds $r$, RC saturation first affects the high dose rate plot which departs from (17) to follow (18). The zero dose rate plot merges with (18) from a higher dose since RC saturation is 
delayed at low dose rate. Taking a larger $r$ value would shift the onset of RC saturation towards higher doses and extend the validity of the direct recombination SSORs over a larger dose range. Of course, direct recombination is recovered up to trap saturation if $r>1$. DRE obtained in conditions of RC saturation consist of ELDRS only, SSORs being always higher at low dose rate. Fig. 5 shows that the ELDRS amplitude is similar to that predicted for direct recombination if $D<r$. It nevertheless affects a much more restricted dose range because DRE vanish upon RC saturation.

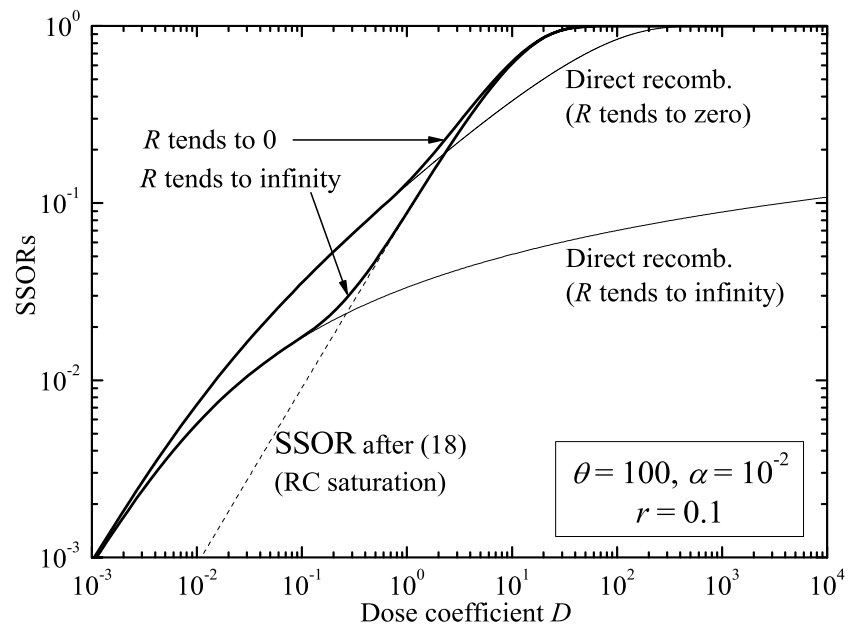

Fig. 5. SSORs at infinite and zero dose rate (solid lines) for $\theta=100$, $\alpha=0.01$ and $r=0.1$. Thin lines show the same limit plots for direct recombination and the dashed line correspond to (18).

\section{Summary and basic lessons}

DRE basically increase with the recombination efficiency, i.e. when $\alpha$ decreases. The common DRE feature inherent in the trapping-recombination competition is an ELDRS. Its magnitude increases as $r$ is raised at fixed $\theta$ and conversely. Enhanced high dose rate sensitivity (EHDRS) occurs when recombination efficiency is limited by a small hole trapping cross-section $(\delta<<\gamma$ or $\theta<<1)$, above a certain dose, and is favored by small $r$ values (more traps than RCs, see Fig. 6). EHDRS is therefore the signature of an indirect recombination process (i.e through a RC center) at least limited by a weak probability of trapping on RCs.

Fig. 6 displays the relative difference $\Delta$ (in \%) between SSORs obtained for $R \rightarrow 0$ and $R \rightarrow+\infty$, respectively, as a function of the dose for $\alpha=10^{-4}, \theta=10^{-2}$ and $r=2,7,20$, and 100 ( $\Delta$ is positive for ELDRS and negative for EHDRS). These plots well illustrate that: (i) ELDRS is not an intrinsic property. Although DRE will depend on the physical nature of $\mathrm{RC}$ (controlling $\delta$ ) and on their concentration $H$, they are also determined by the dose. The critical dose above which ELDRS can turn into EHDRS depends itself on $\alpha, \theta$ and $r$ (Fig. 3 and 6). It cannot be predicted a priori, unless material defects are thoroughly and reliably characterized. This may explain why contradictory DRE behaviors can be measured on materials that are a priori of similar type. As a consequence, accurate DRE characterizations should consider varying the total dose; (ii) as regards the concentration of RC, the crucial parameter is not $H$ but $r=H / N$. The DRE magnitude increases with $r$ and ELDRS prevails at high $r$ for a given $\theta$. Therefore ELDRS exhibited by BJTs should not be explained by the poor quality of the oxide as proposed in [3]. Although lowquality silica most probably presents a high density of $\mathrm{RC}$, it should also have a high density of trapping states and hence a rather small $r$ value. A more reliable explanation is discussed in the conclusion.

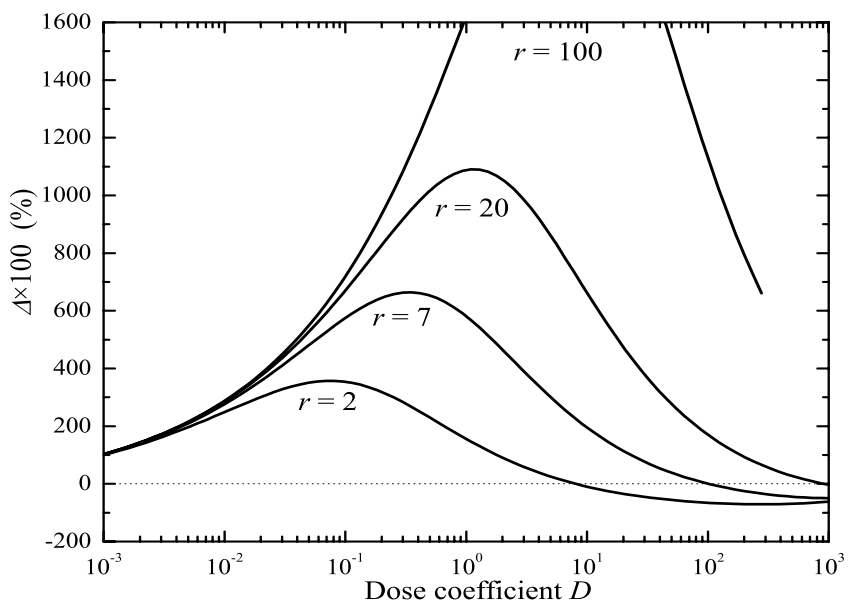

Fig. 6. Relative difference $\Delta$ (in \%) between SSORs obtained for $R \rightarrow 0$ and $R \rightarrow+\infty$ respectively as a function of the reduced dose $D$ for $\alpha=10^{-4}, \theta=10^{-2}$ and $r=2,7,20$, and 100 .

\section{THE CRITICAL DOSE RATE}

A crucial problem is now to identify the critical dose rate $R_{\text {crit }}$ that separates the low dose rate region from the high dose rate one. A good estimate is given by the location of the inflection point of sigmoid-like curves. The latter must be sought numerically, the formal treatment being intractable. For simplicity, we assume that traps share the same cross section $\left(\alpha_{k}=\alpha\right)$. For direct recombination $(\theta>>1$ and $r>>1)$, computed $R_{\text {crit }}$ values are well fitted to $R_{\text {crit }}=0.74 \times \alpha \times \mathrm{D}^{1.07}$ when $D$ and $\alpha$ lie within the interval $10^{-4}$ to 1 . Given that $R=g_{0} / \gamma N^{2}$, the critical dose rate is then independent of $\alpha$. For a generation efficiency $G \sim 5.18 \times 10^{12} \mathrm{EHP} \mathrm{cm} \mathrm{rad}^{-1}$ in silica:

$g_{0 \text { crit }}\left(\operatorname{rad}\left(\mathrm{SiO}_{2}\right) \mathrm{h}^{-1}\right) \approx 5.14 \times 10^{-10} \times \beta \times N^{2} \times D^{1.07}$.

If recombination efficiency is limited by a small $\delta$ or $H$ value, $g_{0 \text { crit }}$ is much smaller than predicted by (19). In situations where EHDRS can arise, i.e. for $\theta \leq 1$, it is found to be inversely proportional to $\alpha=\beta / \gamma$. The lowest value, reached for $\alpha=1$ (negligible DRE for $\alpha>1$ ), is approximately:

$g_{0 \text { crit }}\left(\operatorname{rad}\left(\mathrm{SiO}_{2}\right) \mathrm{h}^{-1}\right) \approx 4.56 \times 10^{-13} \times \beta \times N^{2} \times D^{1.095}$

Critical dose rates generated by this equation are actually among the lowest ones for indirect recombination. They roughly set a lower limit for trap systems. Fig. 7 situates the critical dose rate (20) in regions delimited by reasonable lower and upper order of magnitudes of $\beta$ for electrons, namely $10^{-13}$ $<\beta<10^{-7} \mathrm{~cm}^{3} \mathrm{~s}^{-1}$, for $D=10^{-3}$ and 1 . For densities of traps in the range $10^{15}$ to $10^{18} \mathrm{~cm}^{-3}$, the low dose rate region can extend up to at least 10 to $10^{7} \mathrm{rad} \mathrm{h}^{-1}$ if the total number of created EHP is one thousandth of the density of traps, and up to much greater values at higher doses. 


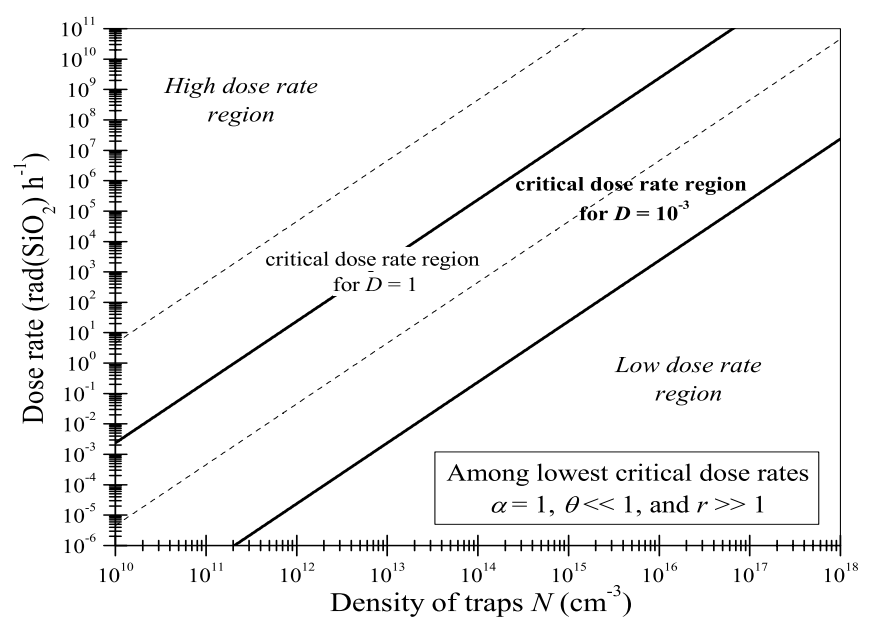

Fig. 7. Typical low dose rate, high dose rate and critical dose rate regions for indirect recombination $(\alpha=1, \theta<<1, r>>1)$ and electron trapping, as delimited according to $10^{-13}<\beta<10^{-7} \mathrm{~cm}^{3} \mathrm{~s}^{-1}$ for $D=10^{-3}$ and $D=1$.

\section{EXAMPLE OF DATA INTERPRETATION FOR OPTICAL FIBERS}

\section{A. Materials}

The trial material consisted of germano-silicate fiber (GSF) samples. Although technological applications would rather be interested in erbium- or ytterbium-doped fibers, this choice is the best to demonstrate the applicability of the model because very well characterized GSF samples are available. Mechanisms of defect formation under radiation exposure have been satisfactorily elucidated in these fibers, even if some controversies remain [10,11]. GSF are the common passive fibers used for signal transmission at $1550 \mathrm{~nm}$. They also serve as host matrix for erbium doping in the standard EDFA technology. In this respect, the results below provide a proper background to assess the effects of erbium addition.

Three types of GSF samples named GeD1, GeD2, and GeD3, produced by iXFiber SAS, France, have been drawn from the same preform at 3 drawing speeds. Composition and opto-geometrical details of the samples are given in [11] with the characterization of their major defects. Radiation-induced optically active centers mainly consist of the so-called Ge(1) and $\mathrm{Ge}(2)$ centers that are formed upon electron trapping $[10,11]$. This directly matches the hypothesis made in the model and for the critical dose rate prediction. The density of radiation-induced $\mathrm{Ge}(1)$ and $\mathrm{Ge}(2)$ centers, i.e. trapped electrons, was measured by electron spin resonance (ESR) as a function of the dose [11]. It was found to saturate above $10^{3}$ $\mathrm{krad}$, at $\sim 3.2 \times 10^{17} \mathrm{~cm}^{-3}$ for $\mathrm{Ge}(1)$ and $\sim 5 \times 10^{17} \mathrm{~cm}^{-3}$ for $\mathrm{Ge}(2)$. The total electron trap density is thus $N \sim 8.2 \times 10^{17} \mathrm{~cm}^{-3}$ at least (additional ESR-silent traps may contribute to $N$ ).

\section{B. Irradiations and SSORs measurements}

Irradiations were performed by 3 different facilities at room temperature (RT). "Low" dose rate irradiations at 30, 100 and $500 \mathrm{rad} \mathrm{h}^{-1}$ were given with gamma rays from a ${ }^{60} \mathrm{Co}$ source. A laboratory copper-anode $\mathrm{x}$-ray tube operating at $45 \mathrm{kV}$ was used for irradiations at $2.2 \times 10^{5}$ and $2.6 \times 10^{6} \mathrm{rad} \mathrm{h}^{-1}$. At least, the Asterix facility of the French Commissariat à l'Energie Atomique (CEA), at Gramat, France, which delivers $1 \mathrm{MeV}$ pulsed $\mathrm{x}$-rays, allowed us to reach dose rates in the range $4.5 \times 10^{13}$ to $1.91 \times 10^{15} \mathrm{rad} \mathrm{h}^{-1}$.
Trap filling was measured by thermally stimulated luminescence (TSL) on irradiated fibers. In this technique, samples are heated up at $1 \mathrm{~K} \mathrm{~s}^{-1}$ from 40 to $600{ }^{\circ} \mathrm{C}$. Trapped carriers are progressively released from traps of increasing depth and the main part of them subsequently recombine with carriers of opposite polarity on RCs. A photo-multiplier tube detects the light emitted upon recombination and a glow curve is obtained by plotting the TSL intensity as a function of temperature. The glow curves we measured from GeD1, 2 and 3 all present a single broad peak around $250{ }^{\circ} \mathrm{C}$, irrespective of the photon energy [9]. As usual in amorphous materials, such a broad peak comprises the superimposition of overlapping elementary peaks related to defects whose energy levels are closely distributed in the gap. The TSL intensity is virtually zero at RT. In agreement with a basic hypothesis of the model, this indicates that traps were thermally stable during irradiation. TSL readouts were found to empty all traps and to result in full bleaching of the samples. We demonstrated in [12] that, in these particular conditions, the glow-curve integrals are directly proportional to the SSORs probed by the TSL readout. Integration was done on the whole of the temperature range so the measured SSORs given below refer to the full collection of traps (global trap filling).

\section{Results and comparison with the model}

Fig. 8 shows the SSORs in GeD2 after $45 \mathrm{kV}$ x-ray irradiations as a function of the dose. Error bars represent a $15 \%$ uncertainty estimated according to the typical accuracy of our dosimetry and TSL measurements. Doses below 15 $\mathrm{krad}$ have been given at $2.2 \times 10^{5} \mathrm{rad} \mathrm{h}^{-1}$. Higher doses were given at $2.6 \times 10^{6} \mathrm{rad} \mathrm{h}^{-1}$. In good agreement with the formation of $\mathrm{Ge}(1)$ and $\mathrm{Ge}(2)$ centers [11], saturation is observed from $\sim 10^{3} \mathrm{krad}$. SSORs on the vertical axis have been scaled by considering that saturation corresponds to $\mathrm{SSOR}=1$.

If $G \sim 5.18 \times 10^{12} \mathrm{EHP} \mathrm{cm} \mathrm{rad}^{-1}$ and $N>8.2 \times 10^{17} \mathrm{~cm}^{-3}$, the dose coefficient $D_{\text {sat }}$ corresponding to $10^{3} \mathrm{krad}$ is at the most 6.3. Far below saturation, at $D=0.063(\sim 10 \mathrm{krad})$, the minimum critical dose rate calculated according to (20) for $\beta=10^{-13} \mathrm{~cm}^{3} \mathrm{~s}^{-1}$ and $N=8.2 \times 10^{17} \mathrm{~cm}^{-3}$ is $\sim 1.5 \times 10^{9} \mathrm{rad} \mathrm{h}^{-1}$. As a consequence, the dose rates used here should fall within the low dose rate region. Accordingly, we investigated possible

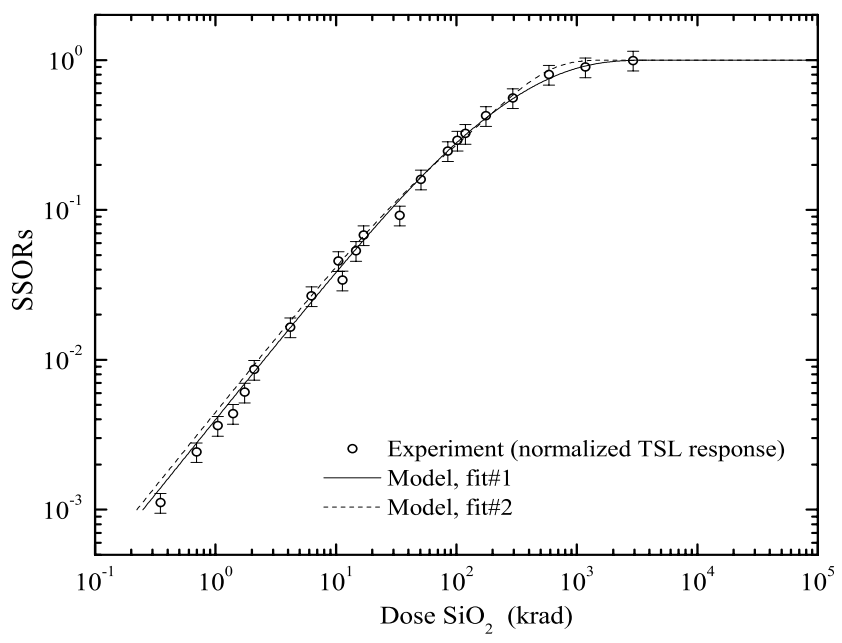

Fig. 8. Dose dependency of the TSL response of GeD2 in terms of SSOR (normalization with respect to the saturation plateau). 
fits of the dose dependency by calculating SSORs at the low dose rate limit. Two of them, referred to as fit\#1 and fit\#2, are shown in Fig. 8. As suggested by Fig. 9 and 10, all fits have been sought by exploring parameters able to produce EHDRS, i.e. for $\theta<<1$. For convenience, a same capture cross section has been taken for all traps (single $\alpha$ ).

In fit\#1, the recombination efficiency is only limited by hole trapping and not by saturation of RCs $(r>>1)$. This fit therefore obeys the formal solution (16). Equation (16) has been fitted to the data of Fig. 8 based on only 2 adjustable variables: the cross-section ratio $\alpha$, and the density of trap $N$. The latter is required to convert the dimensionless dose $D$ into the physical dose in silica. The best fit was obtained for $\alpha=0.4$ and $N=1.29 \times 10^{18} \mathrm{~cm}^{-3}$ ( $N$ is consistent with the minimal value $8.2 \times 10^{17} \mathrm{~cm}^{-3}$ set in [11]). We then computed the dose rate dependency of SSORs by taking $\theta=10^{-5}$ and $r=10^{2}$ to comply with the conditions $\theta<<1$ and $r>>1$. Predicted behaviors are compared to data in Fig. 9 for 10, 50 and $100 \mathrm{krad}$ doses. In addition to $N$ and $\alpha=\beta / \gamma$, the parameter $\beta=10^{-10} \mathrm{~cm}^{3} \mathrm{~s}^{-1}$ was used to convert the dimensionless dose rate $R=g_{0} / \gamma N^{2}$ into the physical one in rad $\mathrm{h}^{-1}$. Except this calibration, no additional parameters were fitted to pass from fit\#1 of Fig. 8 to fits of Fig. 9 (greater $\beta$ values would shift computed plots towards high dose rate).

Error bars in Fig. 9 still correspond to $15 \%$ of the mean value, except for gamma irradiations as explained below. This error only accounts for measurement uncertainties and not for variability between the $\mathrm{GeD} 1, \mathrm{GeD} 2$ and $\mathrm{GeD} 3$ samples. Apart from this variability, the drawing speed does not affect the dose and dose rate dependences. Taking the standard deviation into account would enlarge the error bars.

In Fig. 9, the predicted critical dose rates are about $8 \times 10^{12}$, $5 \times 10^{13}$, and $1 \times 10^{14} \mathrm{rad} \mathrm{h}^{-1}$ at 10,50 , and $100 \mathrm{krad}$ respectively. Data show that trap filling is slightly enhanced at high dose rate and suggest that transitions between the low and high dose rate regimes are well located in a region encompassing these predictions. The model is in qualitative and almost in quantitative agreement with measurements. The main discrepancies appear for gamma irradiations at 10 and $50 \mathrm{krad}$, where measured responses are markedly below the expected asymptote (encircled symbols), and for pulsed $\mathrm{x}$ rays at 100 krad where the EHDRS amplitude is underestimated.

As regard encircled data points, the distance to the expected value is similar at 10 and $50 \mathrm{krad}$. It does not correspond to a dose rate effect since the DRE magnitude should be dose dependent, as seen for the pulsed x-ray points (the EHDRS amplitude is slight, but it increases with the dose). Actually, gamma and x-ray irradiations were not conducted in the same conditions. Gamma irradiations and TSL readouts were separated by more than 2 months whereas the readouts were performed rapidly after exposures for continuous and pulsed $\mathrm{x}$ rays. Although thermal annealing of radiation-induced defects in GeD1-3 is slow at RT, about 5\% in 8 hours [9], larger error bars, roughly estimated to $30 \%$ of the measurement, have been reported for gamma irradiations. At $100 \mathrm{krad}$, data points given by gamma irradiations did not suffer from this problem and match the predicted filling in the low dose rate regime.

To improve the agreement between measured and computed EHDRS amplitude at $100 \mathrm{krad}$, we tried other fit parameters

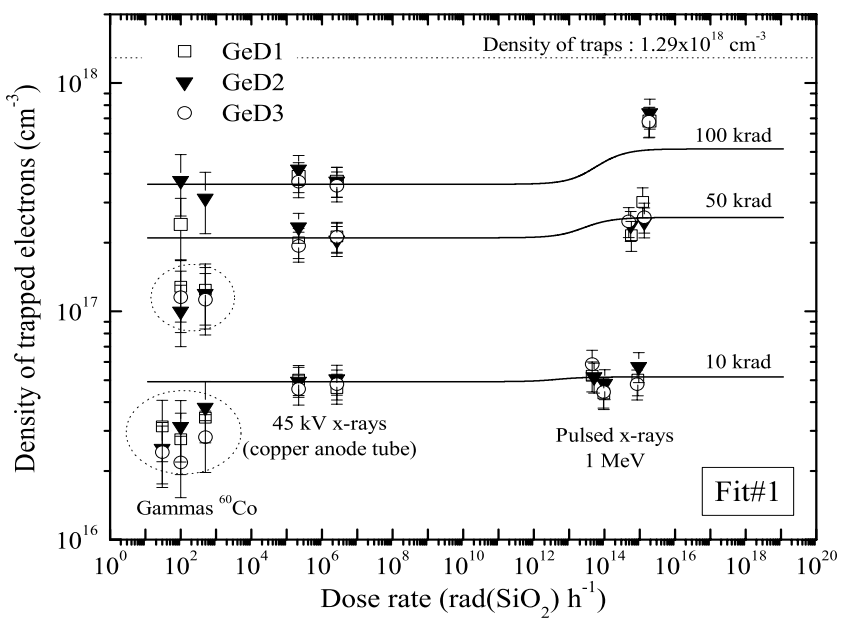

Fig. 9. Steady-sate trap filings in GeD1, GeD2 and GeD3 after 10, 50 and 100 krad doses. Symbols $=$ data. Lines $=$ computed projections for fit\#1. TSL responses have been converted into absolute electron densities with $N=1.29 \times 10^{18} \mathrm{~cm}^{-3}$. Dose rates are calibrated with $\beta=10^{-10} \mathrm{~cm}^{3} \mathrm{~s}^{-1}$.

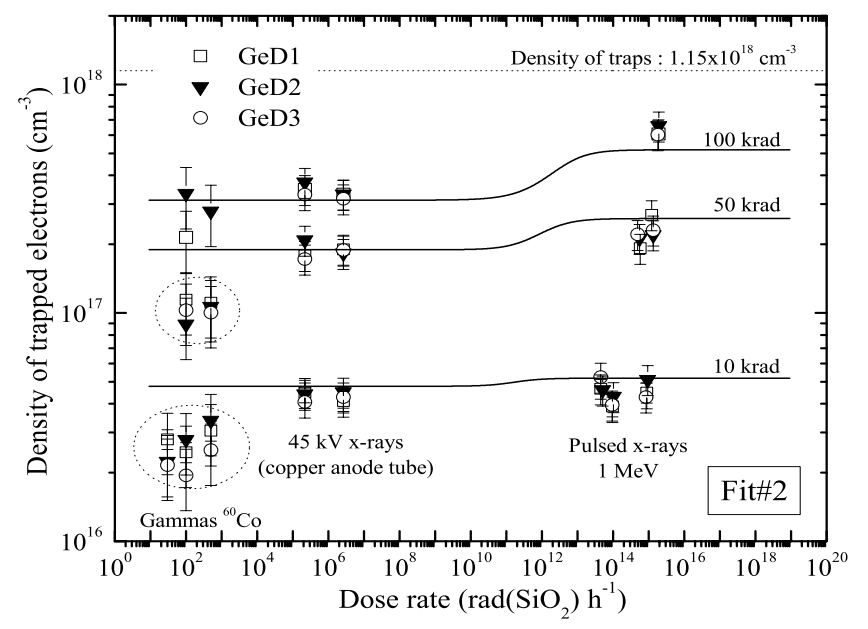

Fig. 10. Same as Fig. 9, except that computed curves were obtained for parameters of fit\#2 (see text). Axes have been scaled with $N=1.15 \times 10^{18} \mathrm{~cm}^{-3}$ and $\beta=10^{-10} \mathrm{~cm}^{3} \mathrm{~s}^{-1}$.

accounting for both dose and dose rate dependences. As expected, increasing the DRE magnitude on the EHDRS side required decreasing $\alpha$ and $r$. Equations could not be resolved analytically so the fitted curves were obtained numerically. A satisfactory result is given by the fit\#2 of Fig. 8 whose parameters also led to the computed curves of Fig. 10 without additional fitting operation. The fitting parameters are now $\theta=10^{-4}, \alpha=0.25, r=0.25$. The best dose scaling (Fig. 8) is obtained for $N=1.15 \times 10^{18} \mathrm{~cm}^{-3}$. In Fig. 10, the dose rate calibration again corresponds to $\beta=10^{-10} \mathrm{~cm}^{3} \mathrm{~s}^{-1}$. The EHDRS amplitude produced by fit\#2 describes the actual one more satisfactorily at $100 \mathrm{krad}$, but the theory now overestimates trap filling at small doses (see also fit\#2 in Fig. 8). Fit\#1 and fit\#2 correctly reproduce the dose and dose rate dependences, each with a single set of a few adjustable parameters having a clear physical meaning. The order of magnitude of the critical dose rate, predicted e.g. from (20) for electron trapping and $\beta=10^{-10} \mathrm{~cm}^{3} \mathrm{~s}^{-1}$, is also confirmed by data of Fig. 9 and 10 . The coefficient $\alpha$ is a few tenths in fit\#1 and fit\#2. This rather "high" value actually prevents the appearance of an ELDRS at low doses. Simulations indeed show that EHDRS is the case at any dose. 


\section{CONCLUSION}

For devices intended for space-based applications, it is crucial to state whether a dose rate extrapolation is required or not after accelerated irradiation tests. The answer depends on the DRE nature and the critical dose rate. Both are controlled by the relevant dimensionless ratios and by the dose. ELDRS constitutes the most adverse situation because degradation will be underestimated by accelerated tests compared with damage produced at typical space dose rate $\left(10^{-2}\right.$ to $\left.1 \mathrm{rad} \mathrm{h}^{-1}\right)$. In addition, the DRE amplitude is often much larger for ELDRS than for EHDRS (Fig. 6). Dose rate extrapolation is also unnecessary if dose rates used in routine tests fall well below the critical value. The latter has been shown to increase almost linearly with the dose and to be proportional to the trapping coefficient $\beta$. It also presents a quadratic dependency on the density of traps $N$. GSF, which e.g. exhibit a slight EHDRS within the 10 to $50 \mathrm{krad}$ range and a critical dose rate above $10^{11} \mathrm{rad} \mathrm{h}^{-1}$, would not require dose rate extrapolation.

Each coefficient $\beta, \gamma$, and $\delta$ is the product of the thermal velocity at the edge of the band by a trapping or recombination cross-section. In amorphous silica, disorder-related localization is weak at the bottom of the $\mathrm{CB}$ and the thermal velocity of electrons is very high, of the order of $10^{7} \mathrm{~cm} \mathrm{~s}^{-1}$ [13]. Localization effects are stronger at the top of the VB where holes move by hopping between localized states. As a result, the intrinsic hole mobility is about $10^{6}$ times smaller than that of electrons at RT $\left(2 \times 10^{-5}\right.$ vs $20 \mathrm{~cm}^{2} \mathrm{~V}^{-1} \mathrm{~s}^{-1}$ respectively [13-15]). Given that the field enhances the drift velocity of holes by lowering the hopping activation energy, the unbiased thermal velocity should be at least $10^{6}$ times smaller for holes than for electrons. This probably explains why we found $\theta<<1$ for both fit\#1 and fit\#2, that is $\delta<<\gamma$. From $\beta=\sigma v_{\text {th }}$ and $v_{\text {th }}=10^{7} \mathrm{~cm} \mathrm{~s}^{-1}$, the electron trapping cross section corresponding to the value $\beta=10^{-10} \mathrm{~cm}^{3} \mathrm{~s}^{-1}$ found in Fig. 9 and 10 , is $\sigma=10^{-17} \mathrm{~cm}^{2}$. This order of magnitude is correct as regards trapping on neutral centers as in GSF [13].

In systems where degradation is due to hole trapping, DRE can be predicted by switching the conduction and valence bands in Fig. 1. Due to the very low thermal velocity of holes, the trapping and recombination coefficients $\beta$ and $\gamma$ should now be very small, and one should accordingly have $\theta>>1$. In these conditions, DRE should consist of ELDRS only. In addition, decreasing $\beta$ by more than 6 orders of magnitude should drop the critical dose rate in similar proportion. According to the values given in Fig. 7 for electron trapping, critical dose rate associated with hole trapping should more likely fall within standard experimental ranges for density of traps into the range $10^{15}$ to $10^{18} \mathrm{~cm}^{-3}$. The clear ELDRS reported in [6,5] for an EDFA irradiated with dose rates between 50 and $1200 \mathrm{rad} \mathrm{h}^{-1}$ thus suggests that the radiation-induced attenuation (RIA) in erbium-doped fibers probably results from the trapping of holes. Note also that BJTs are damaged by hole trapping [1-4] and significant ELDRS is observed at rather standard dose rates. Characterizations of radiation-induced centers in ytterbium- doped silica fibers also showed that traps populated under irradiation and involved in optical losses were hole traps $[7,8]$. Major DRE should therefore be observed in these fibers. We are currently investigating the dose rate dependency of the RIA in erbium and ytterbium-doped fibers in the framework of the present model to confirm its good relevance for optical fibers of technological interest for the aerospace industry.

\section{ACKNOWLEDGMENT}

The authors are indebted to Marc Gaillardin, Claude Marcandella and Philippe Paillet, with CEA, France, for gamma and pulsed $\mathrm{x}$-ray irradiations.

\section{REFERENCES}

[1] R.L. Pease, R.D. Schrimpf, D.M. Fleetwood, "ELDRS in Bipolar Linear Circuits: A Review," IEEE Trans. Nucl. Sci., vol. 56, no. 4, pp. 18941908, 2009

[2] D.M. Fleetwood, "Total Ionizing Dose Effects in MOS and Low-DoseRate-Sensitive Linear Bipolar Devices," IEEE Trans. Nucl. Sci., vol. 60, no. 3, pp. 1706-1730, 2013

[3] J. Boch, F. Saigné, A. D. Touboul, S. Ducret, J.-F. Carlotti, M. Bernard, R. D. Schrimpf, F. Wrobel, G. Sarrabayrouse, "Dose Rate Effects in Bipolar Oxides: Competition Between Trap Filling and Recombination," Appl. Phys. Lett., vol. 88, 232113, 2006

[4] J. Boch, F. Saigné, R.D. Schrimpf, J.-R. Vaillé, L. Dusseau, S. Ducret, M. Bernard, E. Lorfèvre, C. Chatry, "Estimation of Low-Dose-Rate Degradation in Bipolar Linear Integrated Circuits Using Switching Experiments," IEEE Trans. Nucl. Sci., vol. 52, no. 6, pp. 2616-2621, 2005

[5] B. Brichard, A. Fernandez Fernandez, H. Ooms, F. Bergmans, "Gamma Dose Rate Effect in Erbium-Doped Fibers for Space Gyroscopes", in OFS'16:16 th International Conference on Optical Fiber Sensors, Nara City, Japan, 2003, pp. 336-339

[6] O. Gilard, J. Thomas, L. Trousselier, M. Myara, P. Signoret, E. Burov, M. Sotom, "Theoretical Explanation of Enhanced Low Dose Rate Sensitivity in Erbium-Doped Optical Fibers," Appl. Opt., vol. 51, no. 13, pp. 2230-2235, 2012

[7] F. Mady, M. Benabdesselam, Y. Mebrouk, B. Dussardier. (2010, September). Radiation Effects in Ytterbium-Doped Silica Optical Fibers: Traps and Color Centers Related to the Radiation-Induced Optical Losses. Presented at RADECS 2010: International Conference on Radiation Effects on Components and Systems. Paper LN2. [Online]. Available: http://www.radecs2010.ait.ac.at/index.php?link=programme

[8] F. Mady, M. Benabdesselam, W. Blanc, "Thermoluminescence Characterization of Traps Involved in the Photodarkening of YtterbiumDoped Silica Fibers," Opt. Lett., vol. 35, no. 21, pp. 3541-3543, 2010

[9] M. Benabdesselam, F. Mady, S. Girard, "Assessment of Ge-doped Optical Fibre as a TL-Mode Detector," J. Non-Cryst. Sol., vol. 360, pp. 9-12, 2013.

[10] D. L. Griscom, "On the Natures of Radiation-Induced Point Defects in $\mathrm{GeO}_{2}-\mathrm{SiO}_{2}$ Glasses: Reevaluation of a 26-year-old ESR and Optical Data," Opt. Mat. Express, vol. 1, no. 3, pp. 400-412, 2011

[11] A. Alessi, S. Girard, C. Marcandella, S. Agnello, M. Cannas, A. Boukenter, Y. Ouerdane, "X-ray Irradiation Effects on a Multistep GeDoped Silica Fiber Produced Using Different Drawing Conditions," $J$. Non-Cryst. Sol., vol. 357, pp. 1966-1970, 2011.

[12] F. Mady, D. Lapraz, P. Iacconi, "Dimensionless Study of the Total Response of Thermally Stimulated Luminescence," Rad. Meas., vol. 43, pp. $180-184,2008$

[13] H. J. Fitting, J.U. Friemann, "Monte Carlo Studies of the Electron Mobility in $\mathrm{SiO}_{2}$," Phys. Stat. Sol. (a), vol. 69, pp. 349-358, 1982

[14] R. C. Hughes, "Charge-Carrier Transport Phenomena in Amorphous $\mathrm{SiO}_{2}$ : Direct Measurement of the Drift Mobility and Lifetime," Phys. Rev. Lett., vol. 30, pp. 1333-1336, 1973

[15] R. C. Hughes, "Time-Resolved Hole Transport in $a-\mathrm{SiO}_{2}$," Phys. Rev. B, vol. 15 , no. 4, pp. 2012-2020, 1977 